\title{
Trends in Microbiology
}

\section{Forum \\ Black Truffle, a Hermaphrodite with Forced Unisexual Behaviour}

\author{
Marc-André Selosse, ${ }^{1, \star}$ \\ Laure Schneider-Maunoury, ${ }^{1}$ \\ Elisa Taschen, ${ }^{2}$ \\ François Rousset, ${ }^{3}$ and \\ Franck Richard ${ }^{4}$
}

The life cycle of the black truffle (Tuber melanosporum) includes a mating before sporulation: although the species is hermaphroditic, mating turns out to involve parents with very different features, that mostly behave as male or female only, suggesting that this species undergoes forced dioecism.

The black truffle, a fascinating icon of the French gastronomy, turns out to have a no less fascinating sexual life. This soil fungus normally lives in soil as a haploid mycelium that associates with tree roots [1], forming symbiotic organs called mycorrhizae, from which it obtains sugar. The flavoured, edible and prized part of the truffle is an underground fruitbody, containing the meiotic spores (Figure 1). Animals attracted by the odour find and eat the fruitbodies, and the spores are dispersed in the faeces since their thick cell wall protects them from digestion.

The resistance of spores to enzymatic attacks delayed the discovery that two haploid partners are required to build a truffle fruitbody. The DNA extraction protocols used in the first instance revealed a single partner producing the outer skin and internal tissues (the so-called gleba), and because of this, truffles were long considered autogamous [2]. More recently, harsher methods allowed the extraction of spore DNA and identified a second partner that contributes to spore genomes, together with the previous partner [3]. A mating event leads to the formation of a virtual diploid zygote, which thereafter undergoes meiosis to produce the numerous haploid spores (Figure 1). Thus, one parent (considered as the maternal one) provides genes as well as food and protection to the spores, while the other (the paternal parent) provides only genes. Black truffle haploid mycelia are considered to potentially be hermaphrodites, that is, can be mother and father, but autogamy is forbidden by the existence of mating types, a mechanism of self-sterility common among fungi: each haploid individual carries either the MAT11 or the MAT 1-2 allele [4], and mating occurs only between individuals of different mating types, producing MAT1-1/ MAT 1-2 zygotes.

The available black truffle genome [5] prompted the design of microsatellite markers to investigate the distribution of genotypes (genetic individuals) in soil. The first analyses confirmed that the genetic individuals forming the gleba were also present on surrounding mycorrhizae $[6,7]$, establishing evidence for the trophic link between fruitbodies and tree roots by way of the maternal parent. Moreover, labelling of tree photosynthates by ${ }^{13} \mathrm{CO}_{2}$ successfully demonstrated the transfer of carbon from leaves to fruitbodies [8], supporting the nutritional role of maternal individuals. Yet, the spatial analysis of populations, as sampled from mycorrhizae and gleba, revealed two strong structures. First, segregated patches of maternal individuals with identical mating types (i.e., exclusively MAT11 or MAT 1-2) were found in populations. Second, microsatellite analyses revealed a strong isolation by distance among maternal individuals, that is, spatially close individuals are genetically close $[2,7]$. The later feature may relate to the local deposition by animal dispersers of spores issuing from a single or from few fruitbodies, so that immigrant spores are genetically related. However, isolation by distance was observed both in a naturally established truffle field and in plantations of trees inoculated in the nursery [9] where inoculation methods differ from natural conditions by often involving mixes of several fruitbodies. Moreover, immigration of spores from a few fruitbodies does not explain the spatial aggregation of mating types, since spores of a fruitbody are MAT1-1 and MAT 1-2, as the result of meiosis of the MAT1-1/MAT 1-2 zygote. Which then leads to the question: what drives such population structures?

The monitoring of trees in a nursery after inoculation by black truffle spores [6] suggested that although individuals with both mating types coexisted on young seedlings, one of the mating types was progressively excluded over time (Figure 2). The underlying mechanism is not yet fully understood, but a role for vegetative incompatibility has been suggested $[6,10]$. Vegetative incompatibility is a common mechanism in fungi [11], where genetically close individuals reciprocally cooperate, mainly by hyphal fusion and resource sharing. Kinship is ensured by the existence of identical alleles at one or more controlling loci. Vegetative incompatibility protects against exploitation by non-kin, and can exclude competing kinships. In the black truffle, one of the controlling loci would be linked to the mating type (if not the mating type itself [11]), so that the competitive success of one kinship would entail homogeneous MAT1-1 or MAT 1-2 patches. Homogeneity would also be favoured in parts of the genome close to the other putative controlling loci, contributing to the pattern of isolation by distance that emerges in all colonization processes, that is, spontaneous or by anthropic inoculation.

Paternal individuals only recently attracted detailed investigations $[6,9,12]$. Their genotype can be deduced from the zygotic genotype obtained by bulk extraction of spore DNA, by subtracting the maternal genotype as obtained by 


\section{Trends in Microbiology}

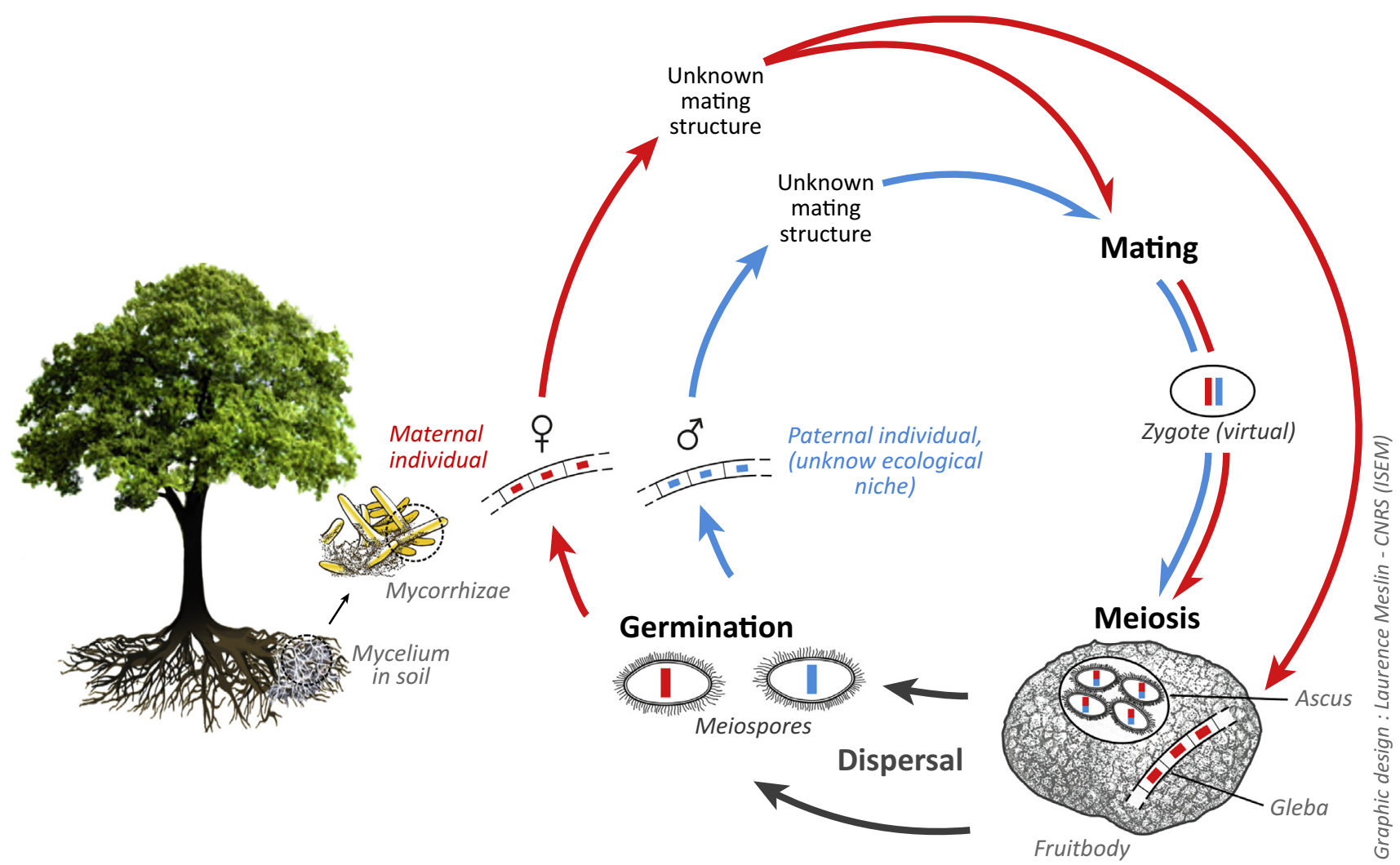

Trends in Microbiology

Figure 1. The Lifecycle of the Black Truffle Tuber melanosporum. The maternal individual is in red; the paternal one in blue. The edible part is a fruitbody made of sterile, nourishing, and protective tissues of maternal origin (the gleba) supporting the development of spores that derive by meiosis of a virtual zygote, itself issuing from a mating event. Each group of spores issuing from one meiosis are embedded by four in one ascus, and fruitbodies contain thousands of asci. Autogamy is prevented by mating types, that is, the maternal individual is MAT 1-1 (or MAT 1-2-1) and the paternal one is MAT 1-2-1 (or, respectively, MAT 1-1). Figure produced with the help of Laurence Meslin, Plateforme Communication Scientifique Visuelle, ISEM.

simple gleba DNA extraction (Figure 1). also genetically close, explaining the conPaternal genotypes revealed two striking sanguinity observed. But where do these features. First, in most fruitbodies, they turned out to be genetically very similar to maternal individuals $[3,9,12]$, significantly more than if mating occurred at random within the population. The load of consanguinity is mainly acting on diploids (due to the accumulation of rare deleterious recessive mutations, hidden by diploidy), but is limited on fully haploid organisms such as the black truffle. But what drives such high inbreeding? It can be explained if paternal genotypes undergo isolation by distance similar to maternal individuals, and if mating does not involve dispersal of male gametes. Under such assumptions, mating occurs between neighbouring individuals that are paternal individuals live in soil? The second finding was the striking differences between fathers and individuals acting as mothers.

Several maternal individuals survive from one year to another; they sometimes produce several neighbouring fruitbodies during several years $[7,9]$, suggesting that their mycelia can reach metric size in soil; and, as stated above, they colonize surrounding tree mycorrhizae $[9,12]$ (Figure 1). By contrast, paternal individuals are very rarely found over 2 successive years and/or in multiple fruitbodies, suggesting much smaller size and shorter lifespan; moreover, they are never found in surrounding mycorrhizae $[6,9,12]$. On the one hand, the latter observation is consistent with the spatial aggregation of mating types on mycorrhizae, with local patches of individuals with a single mating type. On the other hand, this appears at odds with the statement that fathers should be spatially close to mothers and that there is no dispersal of male gametes, in order to explain consanguinity. Thus, paternal individuals occupy another, yet unidentified, niche close to mothers. It has been proposed that germinating spores of the opposite mating type, although not able to settle permanently, may act as fathers $[7,9,10]$; yet other niches in the soil remain to be explored, including short saprotrophic survival: indeed, both mating type alleles 


\section{Dynamics and reproduction of truffle populations}

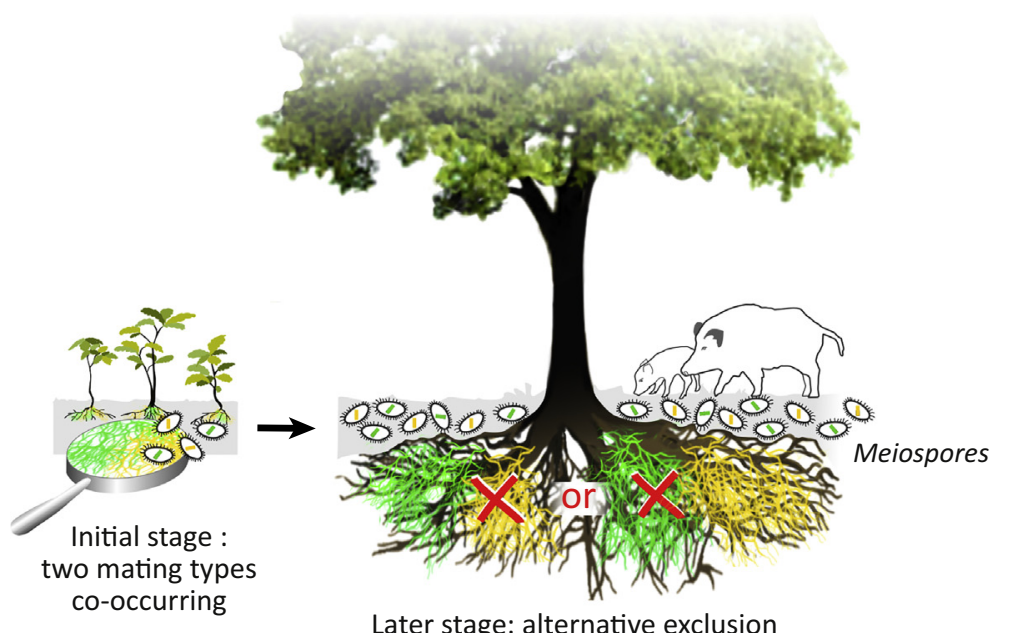

Later stage: alternative exclusion of one mating type

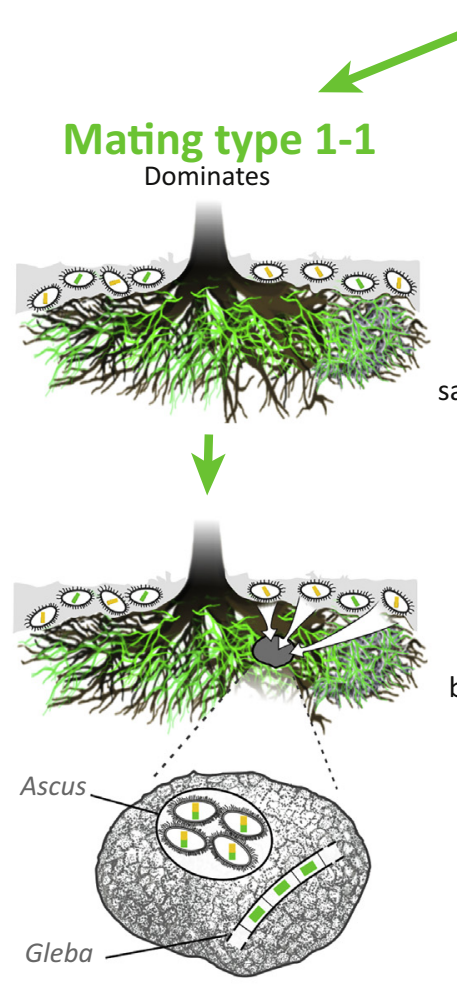

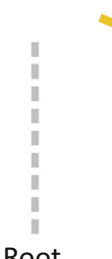

Root colonization by haploid individuals of same mating type

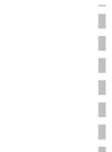

\section{Haploids} of the other mating type behave as males
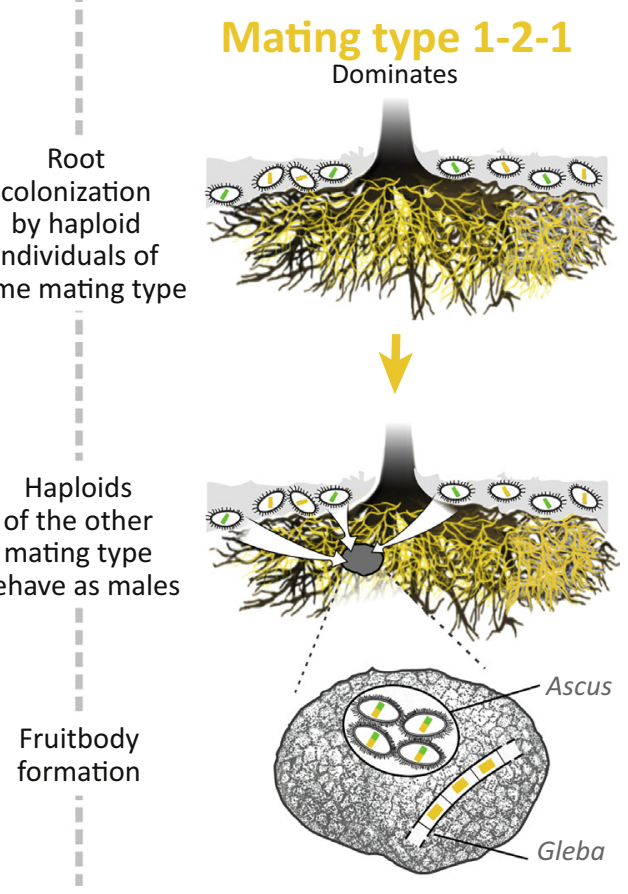

Graphic design : Laurence Meslin - CNRS (ISEM)

Trends in Microbiology

Figure 2. Mating Type Dynamic and Mating in Black Truffle Populations. While at population establishment, haploid individuals of both mating types coexist (top); an exclusion of one type progressively occurs so that mycelia accessing the tree roots locally display the same mating type. Spores also exist in soil, because of spore dispersion, and because of a spore bank issuing from nondispersed fruitbodies. One half of these spores displays the alternative mating type and may provide the paternal contribution. In mating, individuals of the locally dominant mating type behave maternally, that is, also form the gleba supporting fruitbodies. With the help of Laurence Meslin, Plateforme Communication Scientifique Visuelle, ISEM.

can often be detected when extracting soil DNA [6], suggesting that partners of the other mating type do coexist, even if their ecology remains unknown.

Despite potential hermaphroditism, consistent with rare cases where undistinguishable individuals behave as a father in one fruitbody and as mother in other ones [12], most haploid individuals undergo enforced unisexuality (Figure 2). On a given soil patch, individuals of one mating type can establish, form perennial individuals, and support fruitbodies in a maternal way. Although they could (and rarely do [12]) act as fathers as well, they are often too far from compatible partners to be able to provide a maternal contribution. Individuals whose mating type is locally excluded cannot settle, or they remain confined in a more labile niche that remains to be assessed, but they act paternally in mating (Figure 2). This is probably the only contribution they can achieve because of their limited access to resources. Thus, truffle haploid individuals are hermaphrodites enforced to unisexuality, depending on their mating type and that of the surrounding individuals established before their arrival.

\section{Acknowledgments}

We thank three anonymous referees for helpful comments, and apologize that size limitation did not allow us to cite many relevant works.

${ }^{1}$ Institut de Systématique, Évolution, Biodiversité (ISYEB UMR 7205 - CNRS, MNHN, UPMC, EPHE), Muséum national d'Histoire naturelle, Sorbonne Universités, 57 rue Cuvier, 75005, Paris, France

${ }^{2}$ INRA, UMR Eco\&Sols, Place Viala, 34060, Montpellier, France

${ }^{3}$ Institut des Sciences de l'Evolution, Université de Montpellier, CNRS, IRD, EPHE CC 065, Place Eugène Bataillon, 34095 Montpellier cedex 05, France ${ }^{4}$ CEFE UMR 5175, CNRS - Université de Montpellier and Université Paul-Valéry Montpellier - EPHE - 1919 route de Mende, 34293 Montpellier, France

${ }^{\star}$ Correspondence:

ma.selosse@wanadoo.fr (M.-A. Selosse).

http://dx.doi.org/10.1016/j.tim.2017.05.010

References

1. Le Tacon, F. et al. (2015) Certainties and uncertainties about the life cycle of the Périgord black truffle (Tuber melanosporum Vittad.). Ann. For. Sci. 73, 105-117 


\section{Trends in Microbiology}

2. Bertault, G. etal. (2001) Population genetics and dynamics of the black truffle in a man-made truffle field. Heredity 86 , 451-458

3. Riccioni, C. et al. (2008) Tuber melanosporum outcrosses: analysis of the genetic diversity within and among its natural populations under this new scenario. New Phytol. $180,466-478$

4. Rubini, A. et al. (2011) Isolation and characterization of MAT genes in the symbiotic ascomycete Tuber melanosporum. New Phytol. 189, 710-722

5. Martin, F. et al. (2010) Périgord black truffle genome uncovers evolutionary origins and mechanisms of symbiosis. Nature 464, 1033-1038
6. Rubini, A. (2011) Tuber melanosporum: mating type distribution in a natural plantation and dynamics of strains of different mating types on the roots of nursery-inoculated host plants. New Phytol. 189, 723-735

7. Murat, C. et al. (2013) Fine-scale spatial genetic structure of the black truffle (Tuber melanosporum) investigated with neutral microsatellites and functional mating type genes. New Phytol. 199, 176-187

8. Le Tacon, F. et al. (2013) Carbon transfer from the host to Tuber melanosporum mycorrhizas and ascocarps followed using a ${ }^{13} \mathrm{C}$ pulse-labeling technique. PLoS One 8, e64626

9. Taschen, E. et al. (2016) How the truffle got its mate: insights from genetic structure in spontaneous and planted
Mediterranean populations of Tuber melanosporum. Mol. Ecol. 25, 5611-5627

10. Selosse, M.-A. et al. (2013) Do black truffles avoid sexual harassment by linking mating type and vegetative incompatibility? New Phytol. 199, 10-13

11. Saupe, S.J. (2000) Molecular genetics of heterokaryon incompatibility in filamentous ascomycetes. Microbiol. Mol. Biol. Rev. 64, 489-502

12. De la Varga, H. (2017) Five years investigation of female and male genotypes in Périgord black truffle (Tuber melanosporum Vittad.) revealed contrasted reproduction strategies. Environ. Microbiol. Published online March 29, 2017. http://dx.doi.org/10.1111/1462-2920.13735 